\title{
Multiverse Fundamental Equation
}

\author{
Khaled (Moh'd Khalaf) Suleiman Alnobani \\ Chemical Engineering, Jordan University of Science and Technology, Irbid 22110, Jordan
}

\begin{abstract}
A discussion of nature is given. Time is discussed with respect to certain other factors of nature. A potential energy equation is given which holds all relevant information about any system at equilibrium or non-equilibrium state with no restriction on number of phases. A differential form of the fundamental equation is also given, and it gives the amount of energy released if one or more of the variables are changed. The states of different distances in coupled verses are derived. There is no system of measurements outside the human mind, every verse calculates dynamically for itself. Vessel design using sub-verses and gates is discussed. The fundamental equation has important application for human self-development (martial arts).
\end{abstract}

Key words: Time, mass, multiverse.

\section{Nomenclature}

$\begin{array}{ll}\text { constant }_{1}, \text { constant }_{2} & \text { Constant } \\ c & \text { Speed of light }=2.99792458 \times 10^{8} \mathrm{~m} / \mathrm{s} \\ F & \text { Force } \\ K & \text { Kinetic energy } \\ m & \text { Mass } \\ p & \text { Momentum } \\ q & \text { General coordinate system } \\ t & \text { Time } \\ T & \text { Kinetic energy } \\ U & \text { Potential energy field } \\ u & \text { Internal energy } \\ \theta & \text { Angle } \\ \hbar & \text { Physical constant }=1.0546 \times 10^{-34} \mathrm{~J} \cdot \mathrm{s} \\ = & \text { Mathematical operator }\end{array}$

\section{Superscripts}

to

Total

\section{Subscripts}

$\begin{array}{ll}i & \text { Index } \\ \text { rev } & \text { Reversible } \\ 0 & \text { Initial value }\end{array}$

\section{Introduction}

Industry is about change, change of raw materials into products; change of low value to a higher value. The theory and aspects of multiverses are previously

Corresponding author: Khaled (Moh'd Khalaf) Suleiman Alnobani, M.Sc., research field: chemical engineering. established [1-4]. There is no system of measurement units outside the human mind. Also the nature calculates for itself and its results do not accord to human calculations by default. Nature laws are dynamic; that is, how nature responds in effect to the same actions differs with time. It is changed according to probabilities enhanced by actions taken within different verses, e.g., charge relaxation decay at high and low conductivity [5] and type of flow (laminar or turbulent) at high and low Reynold's number. This is a characteristic of a living being; humans can drink hot or ice tea. Invariance is a myth. Transport through space is a property of darkness. It is time to recognize and discover physics of darkness. Studying empty space is more important than studying light, because empty space illuminates dark surfaces. Darkness can hold any light pulse and transmit it. When a mass travels at local light speed it loses its matter characteristics and no longer is a material rather a vacuum pulse.

Alfotooh Almakiyya [6], the great physics and nature behavior book did not include mathematical equations because mathematics did not include, not now and not at the author time a satisfactory logic and physics that can describe the nature encountered within or found in the world (universe).

\section{Results and Discussion}

Time is related to mass change, annihilation and 
creation.

$$
\frac{\left(m-m_{0}\right)}{t}=\dot{m}=\text { constant }
$$

For this term to work it shall have the form:

$$
\frac{\left|m-m_{0}\right|}{t}=\text { constant }
$$

The term $1 / \sqrt{1-\frac{v^{2}}{c^{2}}}$ is involved in calculations; it is called here change factor $(\mathrm{CF})$.

$$
\begin{gathered}
\frac{C F-1}{t} m_{0}=\text { constant } \text { or } \frac{C F-1}{t}=\text { constant }_{1} \\
t=\frac{C F-1}{\text { constant }_{1}}
\end{gathered}
$$

constant $_{1}$ could be treated here as scaling factor and assumed equal one. Then:

$$
t=C F-1
$$

This equation is held with dimensional homogeneity, when $v=0$ then $C F=1$ and $t=0$. When the velocity equals light velocity, time explodes.

The exchange is between two phases. At this stage an important question shall be asked: what is $q$ as applied in this equation? As $\theta$ is the angel between $q$ and $p$.

As the driving force for equilibrium is in the same direction of movement then: $\cos \theta=1$.

Distance travelled is a function of pressure, volume, local speed of light, mass and energy. It is worth to say that sometimes difference of quantities is considered and not the particular value of that quantity. The last equation represents the multiverse representation of the second law of thermodynamics. The $(\Delta m) c^{2}$ is the usual term of energy in special relativity.

It could be concluded that there is no rest condition

$$
q=0
$$

in any physical system (there should be a movement or vibration resulting in displacement from the origin), where $q$ is used in potential energy equation:

$$
U=c^{2}\left(m-m_{0}\right)\left(\frac{\ln q}{\cos \theta}+\frac{C 2}{c^{2}\left(m-m_{0}\right)}+\frac{1}{2}\right)
$$

$$
U=f\left(m, m_{0}, q, \theta, c\right)
$$

Basically this equation contains all information about a system, and this is the energy form. $q, \theta$ and $c$ are intensive variables, while $m$ and $m_{0}$ are extensive variables.

$$
\begin{gathered}
d U=\left(\frac{\partial f}{\partial m}\right)_{m_{0}, q, \theta, c} d m+\left(\frac{\partial f}{\partial m_{0}}\right)_{m, q, \theta, c} d m_{0} \\
+\left(\frac{\partial f}{\partial q}\right)_{m_{0}, m, \theta, c} d q \\
+\left(\frac{\partial f}{\partial \theta}\right)_{m_{0}, q, m, c} d \theta \\
+\left(\frac{\partial f}{\partial c}\right)_{m_{0}, q, \theta, m} d c \\
d U=c^{2}\left(\frac{\ln q}{\cos \theta}+\frac{1}{2}\right) d m-c^{2}\left(\frac{\ln q}{\cos \theta}+\frac{1}{2}\right) d m_{0} \\
+\frac{c^{2}\left(m-m_{0}\right)}{\cos \theta} d \ln q \\
-\frac{c^{2}\left(m-m_{0}\right)(\ln q) \sin \theta}{(\cos \theta)^{2}} d \theta \\
+2 c\left(m-m_{0}\right)\left(\frac{\ln q}{\cos \theta}+1\right) d c
\end{gathered}
$$

This equation gives the required information to describe any system. This equation gives the amount of energy released if one or more of the variables are changed, note that time as an explicit variable is not included, but it is found explicitly in $\ln q$.

$$
\begin{gathered}
\frac{\ln q}{t \cos \theta}=\text { constant }_{2} \\
\ln q=\text { constant }_{2}(t \cos \theta)
\end{gathered}
$$

Considering constant $_{2}$ as scaling factor this leaves the equation as:

$$
\begin{gathered}
\ln q=(t \cos \theta) \\
e^{\ln q}=e^{t \cos \theta}
\end{gathered}
$$

$q=e^{t}$ where $\cos \theta=1$. As $t$ is a state, it is substituted in the equation, it takes the states $\left[0 \pi \frac{\pi}{2} \frac{3}{2} \pi\right]$. Actually there was a typing error (it was $\frac{3 \pi}{2}$ instead of $\left.\frac{2 \pi}{3}\right)$ in writing the time state in Ref. [2]. However, there is no extent for the error as there were no more calculations depending on it. 
$q$ takes the states $[1,23.14,4.81,111.318]$ in Figs. 1 and 2 . When the time state was derived $q$ was taken for a particle motion in a line [2]. It should be emphasized here that there is no system of measurement units outside the human mind.

This means that $\ln q$ is a linear function with time and cosine of the angle between direction of movement and momentum. Knowing that time is a state then what changes this state is position determined by $q_{i}$ which is a general coordinate system.

The work done by a conservative force is

$$
W=-\Delta U
$$

and

$$
d W_{\text {rev }}=-P d V^{t o}
$$

where $-\Delta U$ is the change in the potential energy associated with the force. The negative sign provides the convention that work done against a force field increases potential energy, while work done by the

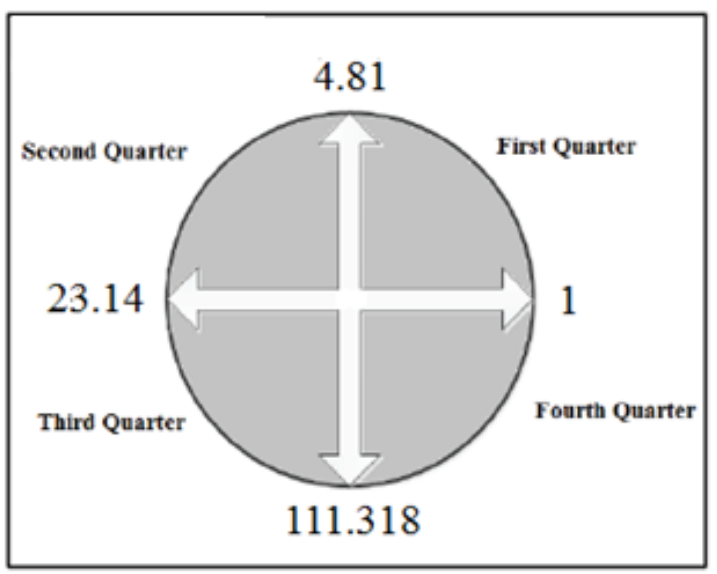

Fig. 1 q state quarters.

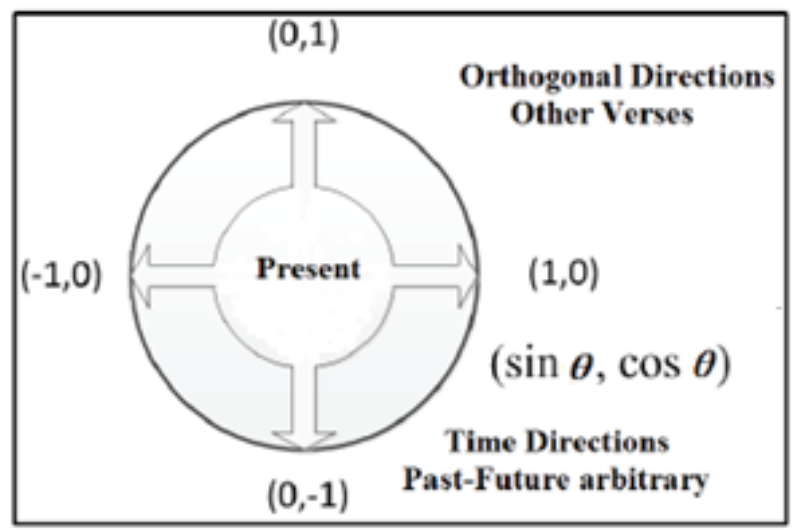

Fig. 2 Time directions. force field decreases potential energy.

$$
d W_{\text {rev }}=d u^{t o}-T d S^{t o}
$$

There are no restrictions on number of phases in this equation. Also, $U$ here is defined also for none equilibrium states. The system is any moving object. There is no need for other energy transformations from the above equation as all the variables are measurable. Legendre transformations are not required here. $q$ is displacement but what is $\ln q$, and what it means. The natural logarithm is an operator working on $q$, but in this weird world how this operator works. It is found in all potential energy terms. There is no zero distance or even close to zero distance. What concerns now is the exchange between verses and how it is related to this operator. What if it is looked to $q$ as displacement and $\ln q$ as distance. Path and none path terms will be packed in one term, then different substitutions will be recognized, which is permitted by the equal operator. As displacement will be converted to updated distance in $\ln q$, a different value is used. It is like a rebuke cube. The current verse is a scrambled cube; there is an algorithm of a certain number of movements that lead to transfer to other organized verses. However, there is a need for an energy representation useful for reactions. Until now the concern with multiverse is with motion and transfer from verse to another verse, in other words "energy". "But what about reactions and matter behavior, like change of state, because it is essential for the future of industries?" Also, what about temperature and pressure? The simple relations for simple systems and restricted conditions of thermodynamics are not acknowledged here.

Recall that transfer between verses happens when energy or any other conservation law is violated and there is a detainment of mass conservation between verses. Also take into account that the process between verses or change is not a result of an arrow of time. A whole new chemical and physical reaction principle is considered and the future of industry virtually must change. Factories will be built on edges between verses. Sub-verses and gates of transfer between verses will be 
the future reactors.

Energy of bonding molecules is found in the potential energy concerning changes in mass.

\subsection{Vessel Design}

Because of the properties of distance and displacement, uses ln q (never could be zero) as the appearing term in the correlations. The wall material of the vessel never affects or is affected by the internal matter and its properties. Therefore the wall material depends only on the outside environment. Extreme temperatures and pressures could be hold within the human hand without affecting or feeling the situation, with no risk at all. In the ultimate situation when the control of all process is optimum, no vessel is needed because there is no contact between the two sides, neither the process side nor the environment side. The vessel is considered a mechanical (no choice inside) sub-verse. To exchange matter between the vessel and the surrounding a gate shall be coded.

\section{Conclusions}

Thermodynamics is related to changes and not to absolute values of state properties, the equations in this paper are related to both changes and reference states or real basic absolute properties. To bring alive the equations in this paper a lot of work should be done about energy lost and gained as mass in annihilation and creation, also an understanding of the displacement term $q$ should be visualized. The speed of light is a very important factor but when it is considered a variable and when it is not, this is a very important factor.

A question should be answered is that "Is there a limit for elementary particles?" The answer is very important and it is philosophical more than physical. A particle of any mass could be annihilated or created. To which verse these particles belong is another issue. Although exchange between verses is permitted, large quantities can affect any system found in it. The equations presented in this paper are the future of calculations held for systems open or closed in a universe. From the terms in the potential energy equation, it could be concluded that there is no limit of energy that could be withdrawn from any object.

As there is no system of measurement units outside the human mind this leaves all our physics, chemistry, mathematics and every concept related, irrelevant. This is more likely to be true because of the life feature of the universe.

As $t$ and $q$ are states variables, then the state is changing with time (dynamic).

The theory entitled here resolves all issues of change, with all its disciplines. No matter what is your mass or where you are you can hold an enormous amount of energy (power and force), this is very important in martial arts styles development, there are new insights for body mass (sub-verse), space, time interactions; even for movement from rest. A close look should be given to what increases and what decreases potential energy in the fundamental equation ${ }^{1}$. Also this thesis has important application in some aspects of black-magic treasures safe design (vessel design).

It should be emphasized here that the fundamental equation form strengthens the path of its derivation because all of the change terms have the same driving force, which is: $\left(m-m_{0}\right)$, which the release of the potential energy depends on.

\section{References}

[1] Alnobani, K. S. 2016. “A New Energy Concept.” Journal of Energy and Power Engineering 10 (2): 116-20.

[2] Alnobani, K. S. 2017. "Multiverse and the Time State." Journal of Energy and Power Engineering 11 (3): 160-3.

[3] Alnobani, K. S. 2017. "Multiverse Spacetime." Journal of Energy and Power Engineering 11 (9): 584-8.

[4] Alnobani, K. S. 2018. "Multiverse Theory." Journal of Energy and Power Engineering 12: 186-91.

[5] Johnston, C. L., and Lazaris, J. 1991. Plane Trigonometry: A New Approach. Prentice Hall, p. 247.

[6] Arabi, I. 1238. "The Introduction." In Alfotooh Almakiyya.

\footnotetext{
${ }^{1}$ The author of this paper is a holder of the Dan-Grade, Taekwondo.
} 